\title{
Rational Equiangular Polygons
}

\author{
Marius Munteanu, Laura Munteanu \\ Department of Mathematics, Computer Science and Statistics, State University of New York, Oneonta, USA \\ Email: Marius.Munteanu@oneonta.edu, Laura.Munteanu@oneonta.edu
}

Received August 3, 2013; revised September 3, 2013; accepted September 10, 2013

Copyright (C) 2013 Marius Munteanu, Laura Munteanu. This is an open access article distributed under the Creative Commons Attribution License, which permits unrestricted use, distribution, and reproduction in any medium, provided the original work is properly cited.

\begin{abstract}
The main purpose of this note is to investigate equiangular polygons with rational edges. When the number of edges is the power of a prime, we determine simple, necessary and sufficient conditions for the existence of such polygons. As special cases of our investigations, we settle two conjectures involving arithmetic polygons.
\end{abstract}

Keywords: Equiangular Polygon; Arithmetic Polygon

\section{Introduction}

A simple way of extending the class of regular polygons is to maintain the congruence of vertex angles while no longer requiring that the edges be congruent. In this generality, the newly obtained equiangular polygons are not all that interesting given one can find plenty of such (nonsimilar) polygons with a given number of edges. Indeed, drawing a parallel line to one of the edges of a regular polygon through an arbitrary point on an adjacent edge yields a trapezoid and a new equiangular polygon with the same number of edges as the initial one (see Figure 1). However, if we also require that all edge lengths be rational numbers and that at least two of these numbers be different (thus excluding regular polygons), in general, such equiangular polygons may not even exist. For example, if we start with the regular pentagon $P_{1} P_{2} P_{3} P_{4} P_{5}$ and draw the parallel $\overline{Q_{1} Q_{2}}$ to $\overline{P_{1} P_{2}}$ as in Figure 1 and if $P_{1} P_{2}$ and $P_{1} Q_{1}$ are rational numbers then, except for $Q_{1} Q_{2}$, all edge lengths of the equiangular pentagon $Q_{1} Q_{2} P_{3} P_{4} P_{5}$ are rational. However, $Q_{1} Q_{2}=P_{1} P_{2}+2 P_{1} Q_{1} \cos (2 \pi / 5)$ is irrational. While this, by no means, proves that equiangular pentagons with

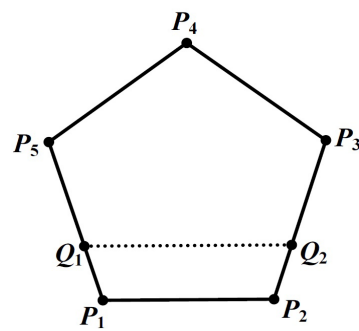

Figure 1. New equiangular pentagons from old. rational edges must be regular, it gives some credibility to the non-existence claim above.

An interesting investigation of equiangular polygons with integer sides is provided in [1], where the author considers the problem of tiling these polygons with either regular polygons or other pattern blocks of integer sides. In particular, he points out that every equiangular hexagon with integer sides can be tiled by a set of congruent equilateral triangles, also of integer sides, and also proposes a general tiling conjecture with an extended tiling set. On the other hand, if one no longer requires integer edges but asks that the vertices be integer lattice points, the only equiangular polygons that will do are squares and octagons (see [2,3]).

Further restricting the class of equiangular polygons with integer sides, in [4], R. Dawson considers the class of arithmetic polygons, i.e., equiangular polygons whose edge lengths form an arithmetic sequence (upon a suitable rearrangement) and shows that the existence of arithmetic $n$-gons is equivalent to that of equiangular $n$-gons whose side lengths form a permutation of the set $\{1,2, \cdots, n\}$. In addition, some interesting existence as well as non-existence results are obtained, but the classification problem for arithmetic polygons with an arbitrary number of edges is left open.

In this note, we address the more general problem of determining all equiangular polygons with rational edges and, as a special case, we settle the classification problem above.

\section{Preliminaries}

First, we derive a necessary and sufficient condition for 
the existence of closed polygonal paths in terms of edge lengths and angle measures.

Proposition 1 Let $l_{1}, l_{2}, \cdots, l_{n}$ and $\theta_{1}, \theta_{2}, \cdots, \theta_{n}$ be positive real numbers with $\theta_{j}<2 \pi, 1 \leq j \leq n$. There exists a closed polygonal path $P_{1} P_{2} \cdots P_{n}$ (with

$P_{1}-P_{2}-\cdots-P_{n}-P_{1}$ oriented counterclockwise) having edge lengths $P_{1} P_{2}=l_{1}, P_{2} P_{3}=l_{2}, \cdots, P_{n} P_{1}=l_{n}$ and the measures of the angles formed by $\boldsymbol{P}_{\mathbf{1}} \boldsymbol{P}_{2}$ with $\boldsymbol{P}_{\mathbf{1}} \boldsymbol{P}_{n}$, $\boldsymbol{P}_{\mathbf{2}} \boldsymbol{P}_{\mathbf{3}}$ with $\boldsymbol{P}_{\mathbf{2}} \boldsymbol{P}_{\mathbf{1}}, \quad \boldsymbol{P}_{\boldsymbol{n}} \boldsymbol{P}_{\mathbf{1}}$ with $\boldsymbol{P}_{n} \boldsymbol{P}_{n-\mathbf{1}}$ equal to $\theta_{1}, \theta_{2}, \cdots, \theta_{n}$, respectively, if and only if

$$
l_{1} \mathrm{e}^{-i \theta_{1}}-l_{2} \mathrm{e}^{-i\left(\theta_{1}+\theta_{2}\right)}+\cdots+(-1)^{n-1} l_{n} \mathrm{e}^{-i\left(\theta_{1}+\cdots+\theta_{n}\right)}=0
$$

and

$$
\theta_{1}+\theta_{2}+\cdots+\theta_{n}=(n-2 k) \pi,
$$

for some integer $k$.

Proof Assuming that such a polygon exists, let $z_{j}$ be the complex number associated to $P_{j}, 1 \leq j \leq n$. As the vector $\boldsymbol{P}_{1} \boldsymbol{P}_{2}$ is the $l_{1} / l_{n}$-multiple of the rotation of $\boldsymbol{P}_{\mathbf{1}} \boldsymbol{P}_{\boldsymbol{n}}$ in $P_{1}$ through $2 \pi-\theta_{1}$ (see Figure 2), we have

$$
z_{2}-z_{1}=\mathrm{e}^{-i \theta_{1}}\left(z_{n}-z_{1}\right) \frac{l_{1}}{l_{n}} .
$$

Based on the same type of argument, regardless of the orientation of triangles $P_{j} P_{j+1} P_{j+2}, 1 \leq j \leq n$, we have

$$
\begin{aligned}
& z_{2}-z_{1}=-\mathrm{e}^{-i \theta_{1}}\left(z_{1}-z_{n}\right) \frac{l_{1}}{l_{n}}, \\
& z_{3}-z_{2}=-\mathrm{e}^{-i \theta_{2}}\left(z_{2}-z_{1}\right) \frac{l_{2}}{l_{1}}=(-1)^{2} \mathrm{e}^{-i\left(\theta_{1}+\theta_{2}\right)}\left(z_{1}-z_{n}\right) \frac{l_{2}}{l_{n}}, \\
& \vdots \\
& z_{n}-z_{n-1}=-\mathrm{e}^{-i \theta_{n-1}}\left(z_{n-1}-z_{n-2}\right) \frac{l_{n}-1}{l_{n-2}} \\
& =\cdots=(-1)^{n-1} \mathrm{e}^{-i\left(\theta_{1}+\cdots+\theta_{n-1}\right)}\left(z_{1}-z_{n}\right) \frac{l_{n-1}}{l_{n}}, \\
& z_{1}-z_{n}=-\mathrm{e}^{-i \theta_{n}}\left(z_{n}-z_{n-1}\right) \frac{l_{n}}{l_{n-1}} \\
& =\cdots=(-1)^{n} \mathrm{e}^{-i\left(\theta_{1}+\cdots+\theta_{n}\right)}\left(z_{1}-z_{n}\right) .
\end{aligned}
$$

Combining these relations with

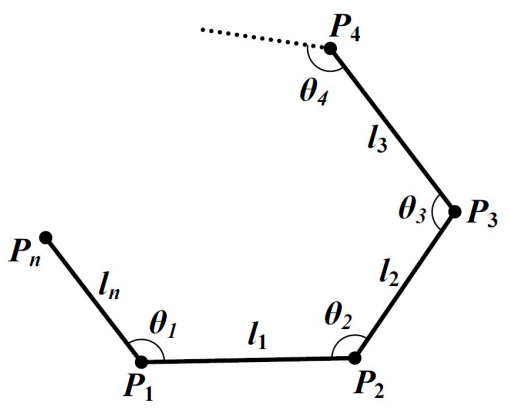

Figure 2. The polygon $P_{1} P_{2} \cdots P_{n}$.

$$
\left(z_{2}-z_{1}\right)+\left(z_{3}-z_{2}\right)+\cdots+\left(z_{1}-z_{n}\right)=0
$$

yields

$$
\begin{aligned}
& -\mathrm{e}^{-i \theta_{1}}\left(z_{1}-z_{n}\right) \frac{l_{1}}{l_{n}}+(-1)^{2} \mathrm{e}^{-i\left(\theta_{1}+\theta_{2}\right)}\left(z_{1}-z_{n}\right) \frac{l_{2}}{l_{n}} \\
& +\cdots+(-1)^{n} \mathrm{e}^{-i\left(\theta_{1}+\cdots+\theta_{n}\right)}\left(z_{1}-z_{n}\right) \frac{l_{n}}{l_{n}}=0,
\end{aligned}
$$

thus proving relation (1.1) from the conclusion. Relation (1.2) follows easily as a consequence of the last relation in the set of $n$ relations above.

Conversely, to prove the existence of a closed polygonal path with given $l_{j}, \theta_{j}, 1 \leq j \leq n$, satisfying (1.1), observe that, starting with an arbitrary point $P_{1}$, we can always consider the points $P_{2}, \cdots, P_{n}$ such that, with the exception of $P_{1} P_{n}$ and the measure of the angles formed by $\boldsymbol{P}_{\mathbf{1}} \boldsymbol{P}_{\mathbf{2}}$ with $\boldsymbol{P}_{\mathbf{1}} \boldsymbol{P}_{n}$ and $\boldsymbol{P}_{\boldsymbol{n}} \boldsymbol{P}_{\mathbf{1}}$ with $\boldsymbol{P}_{\boldsymbol{n}} \boldsymbol{P}_{\boldsymbol{n}-\mathbf{1}}$, all edge lengths and angle measures are as needed. We will prove that the closed polygonal path $P_{1} P_{2} \cdots P_{n}$ satisfies the requirements. To do so, if we let $P_{1} P_{n}=l_{n}^{\prime}$ and denote the measure of the angle formed by $\boldsymbol{P}_{\mathbf{1}} \boldsymbol{P}_{\mathbf{2}}$ with $\boldsymbol{P}_{\mathbf{1}} \boldsymbol{P}_{\boldsymbol{n}}$ by $\theta_{1}^{\prime}$ and $\boldsymbol{P}_{\boldsymbol{n}} \boldsymbol{P}_{\mathbf{1}}$ with $\boldsymbol{P}_{\boldsymbol{n}} \boldsymbol{P}_{\boldsymbol{n}-\mathbf{1}}$ by $\theta_{n}^{\prime}$ then, we need to show that $l_{n}=l_{n}^{\prime}, \theta_{n}=\theta_{n}^{\prime}$, and $\theta_{1}=\theta_{1}^{\prime}$. By applying the direct implication to our polygonal path (with edge lengths $l_{1}, l_{2}, \cdots, l_{n-1}, l_{n}^{\prime}$ and angle measures $\left.\theta_{1}^{\prime}, \theta_{2}, \cdots, \theta_{n-1}, \theta_{n}^{\prime}\right)$, we have

$$
l_{1} \mathrm{e}^{-i \theta_{1}^{\prime}}-l_{2} \mathrm{e}^{-i\left(\theta_{1}^{\prime}+\theta_{2}\right)}+\cdots+(-1)^{n-1} l_{n}^{\prime} \mathrm{e}^{-i\left(\theta_{1}^{\prime}+\cdots+\theta_{n}^{\prime}\right)}=0 .
$$

Equivalently,

$$
l_{n}^{\prime}=(-1)^{n} l_{1} \mathrm{e}^{i\left(\theta_{2}+\cdots+\theta_{n}^{\prime}\right)}+(-1)^{n-1} l_{2} \mathrm{e}^{i\left(\theta_{3}+\theta_{n}^{\prime}\right)}+\cdots+l_{n-1} \mathrm{e}^{i \theta_{n}^{\prime}} .
$$

By factoring out $\mathrm{e}^{i \theta_{n}^{\prime}}$ and applying the modulus on both sides of the equality above, we have

$$
l_{n}^{\prime}=\left|(-1)^{n} l_{1} \mathrm{e}^{i\left(\theta_{2}+\cdots+\theta_{n-1}\right)}+(-1)^{n-1} l_{2} \mathrm{e}^{i\left(\theta_{3}+\cdots+\theta_{n-1}\right)}+\cdots+l_{n-1}\right| .
$$

However, the same type of operations can also be applied to the relation in our hypothesis (involving $\left.l_{1}, l_{2}, \cdots, l_{n}, \quad \theta_{1}, \theta_{2}, \cdots, \theta_{n}\right)$ to obtain

$$
l_{n}=\left|(-1) l_{1} \mathrm{e}^{i\left(\theta_{2}+\cdots+\theta_{n-1}\right)}+(-1)^{n-1} l_{2} \mathrm{e}^{i\left(\theta_{3}+\cdots+\theta_{n-1}\right)}+\cdots+l_{n-1}\right| .
$$

But then $l_{n}=l_{n}^{\prime}$ based on the two formulas above. Now, factoring out $\mathrm{e}^{i \theta_{n}^{\prime}}$ and replacing $l_{n}^{\prime}$ by $l_{n}$ in (1.3), we have

$$
\begin{aligned}
l_{n}=\mathrm{e}^{-i \theta_{n}^{\prime}} & \left((-1)^{n} l_{1} \mathrm{e}^{i\left(\theta_{2}+\cdots+\theta_{n-1}\right)} \cdot\right. \\
& \left.+(-1)^{n-1} l_{2} \mathrm{e}^{i\left(\theta_{3}+\cdots+\theta_{n-1}\right)}+\cdots+l_{n-1}\right)
\end{aligned}
$$

But we also have

*All angles are measured counterclockwise from the first to the second referenced ray. 


$$
\begin{aligned}
l_{n}=\mathrm{e}^{-i \theta_{n}} & \left((-1)^{n} l_{1} \mathrm{e}^{i\left(\theta_{2}+\cdots+\theta_{n-1}\right)} .\right. \\
& \left.+(-1)^{n-1} l_{2} \mathrm{e}^{i\left(\theta_{3}+\cdots+\theta_{n-1}\right)}+\cdots+l_{n-1}\right)
\end{aligned}
$$

Comparing relations (1.4) and (1.5), it follows that $\theta_{n}=\theta_{n}^{\prime}$. To show that $\theta_{1}=\theta_{1}^{\prime}$, let's note that relation (1.2) applied to $P_{1} P_{2} \cdots P_{n}$ implies

$$
\theta_{1}^{\prime}+\theta_{2}+\cdots+\theta_{n}=\left(n-2 k^{\prime}\right) \pi,
$$

for some integer $k^{\prime}$. By hypothesis,

$$
\theta_{1}+\theta_{2}+\cdots+\theta_{n}=(n-2 k) \pi .
$$

Combining the two relations above finishes the proof.

\section{Equiangular Polygons}

If we consider a convex equiangular $n$-gon, then, with notations as in the previous section, we have $\theta_{1}=\theta_{2}=\theta_{n}=\frac{(n-2) \pi}{n}$. In addition, if we let $\xi_{n}=\mathrm{e}^{\frac{2 \pi i}{n}}$, then, based on Proposition 1, we obtain

Theorem 1 Given $l_{1}, l_{2}, \cdots, l_{n} \in \mathbb{R}^{+}$, there exists $a$ convex equiangular $n$-gon with side lengths $l_{1}, l_{2}, \cdots, l_{n}$ (listed counterclockwise) iff

$$
l_{1}+l_{2} \xi_{n}+l_{3} \xi_{n}^{2}+\cdots+l_{n} \xi_{n}^{n-1}=0 .
$$

Definition 1 A rational polygon is a polygon all of whose edge lengths are rational number.

Observation 1 The edges of a non-convex equiangular polygon can be rearranged to form a convex equiangular polygon, so we will only concentrate on the latter.

As a consequence of Theorem 1 , we obtain

Proposition 2 Let $\xi_{n}=\mathrm{e}^{\frac{2 \pi i}{n}}$ and let $N=\phi(n)$ be the degree of the cyclotomic polynomial $\Phi_{n}(X)$. There exists a convex, rational, equiangular n-gon with edge lengths $l_{1}, l_{2}, \cdots, l_{n}$ (ordered counterclockwise) iff the following equalities are satisfied:

$$
\begin{aligned}
& l_{1}+\sum_{j=N+1}^{n} l_{j} a_{1, j-1}=0, \\
& l_{2}+\sum_{j=N+1}^{n} l_{j} a_{2, j-1}=0, \\
& l_{N}+\sum_{j=N+1}^{n} l_{j} a_{N, j-1}=0,
\end{aligned}
$$

where $a_{i, j}$ are defined by

$$
\xi_{n}^{j}=\sum_{i=0}^{N-1} a_{i+1, j} \xi_{n}^{i} .
$$

for all $N \leq j \leq n-1,0 \leq i \leq N-1$.

Proof Let us first note that the definition of $a_{i j}$ makes sense. Indeed, since $\left\{1, \xi_{n}, \xi_{n}^{2}, \cdots, \xi_{n}^{N-1}\right\}$ forms a basis of $\mathbb{Q}\left(\xi_{n}\right)$, for a fixed $j, N \leq j \leq n$, we can define $a_{i, j}$ to be the coefficients of $\xi_{n}^{j}$ in this basis.

For each $N \leq j \leq n-1$, if we replace $\xi_{n}^{j}$ in the equality from Theorem 1 by $\sum_{i=0}^{N-1} a_{i+1, j} \xi_{n}^{i}$, we obtain

$$
\begin{aligned}
& l_{1}+l_{2} \xi_{n}+\cdots+l_{N} \xi_{n}^{N-1}+l_{N+1}\left(\sum_{i=0}^{N-1} a_{i+1, N} \xi_{n}^{i}\right) \\
& +\cdots+l_{n}\left(\sum_{i=0}^{N-1} a_{i+1, n} \xi_{n}^{i}\right)=0 .
\end{aligned}
$$

By reorganizing the terms, the formula above becomes

$$
\begin{aligned}
& \left(l_{1}+\sum_{j=N+1}^{n} l_{j} a_{1, j-1}\right)+\left(l_{2}+\sum_{j=N+1}^{n} l_{j} a_{2, j-1}\right) \xi_{n} \\
& +\cdots+\left(l_{N}+\sum_{j=N+1}^{n} l_{j} a_{N, j-1}\right) \xi_{n}^{N-1}=0 .
\end{aligned}
$$

But then we get a polynomial of degree $N-1$ with rational coefficients having $\xi_{n}$ as a root. This is only possible iff all the coefficients are zero, thus proving the proposition.

Observation 2 By fixing $l_{1}, l_{2}, \cdots, l_{N-1} \in \mathbb{Q}^{+}$, the conditions in the proposition above generate a system of equations

$$
\sum_{j=N+1}^{n} a_{s, j-1} l_{j}=-l_{s}, 1 \leq s \leq N
$$

with $N$ equations and $n-N$ variables $l_{N+1}, \cdots, l_{n}$. Comparing the number of equations and the number of variables, we obtain three cases depending on whether $N=\phi(n)<n-N, \phi(n)=n-\phi(n)$, or $\phi(n)>n-\phi(n)$.

To better understand the three cases above, we have

Lemma 1 For any positive integer $n$, we have the following

1) $n<2 \phi(n)$ iff $n=p^{k}$ for some odd prime $p$ and some positive integer $k$.

2) $n=2 \phi(n)$ iff $n=2^{k}$ for some positive integer $k$.

3) $n>2 \phi(n)$ iff $n=2^{k} m$, where the nonnegative integer $k$ and the odd integer $m, m \geq 3$, are such that either $k \geq 1$ or, if $k=0$, then $m$ is the product of the powers of at least two distinct primes.

Proof Since the third case is the complement of the first two, it is enough to prove the first two cases. So let $n=p_{1}^{k_{1}} p_{2}^{k_{2}} \cdots p_{r}^{k_{r}}$, where $p_{1}, p_{2}, \cdots, p_{r}$ are distinct primes and $k_{i}$ are nonnegative integers.

To prove (1), observe that the inequality $n<2 \phi(n)$ is equivalent to

$$
p_{1}^{k_{1}} p_{2}^{k_{2}} \cdots p_{r}^{k_{r}}<2 p_{1}^{k_{1}-1}\left(p_{1}-1\right) p_{2}^{k_{2}-1}\left(p_{2}-1\right) \cdots p_{r}^{k_{r}-1},
$$

or $p_{1} p_{2} \cdots p_{r}<2\left(p_{1}-1\right)\left(p_{2}-1\right) \cdots\left(p_{r}-1\right)$. To show that $n$ has the desired form, let us assume by contradiction that $r \geq 2$. But then, since $p_{1} \geq 2$ and $p_{2} \geq 3$, 
we have $\left(p_{1}+2\right)\left(p_{2}+2\right)>6$, or equivalently $p_{1} p_{2}+2 p_{2}+2 p_{2}-2>0$. This implies $p_{1} p_{2}>2 p_{1} p_{2}-2 p_{1}-2 p_{2}+2=2\left(p_{1}-1\right)\left(p_{2}-1\right)$.

Together with $p_{i}>p_{i}-1,3 \leq i \leq r$, the second inequality above yields $n>2 \phi(n)$, which contradicts the hypothesis. Thus $r=1$. But then we must also have $p_{1}>2$ since otherwise $n=2^{k_{1}}=2 \cdot 2^{k_{1}-1}=2 \phi(n)$. Conversely, it is easy to see that if $n=p^{k}, p>2$, then $n<2 \phi(n)$.

For (2), by considerations similar to the ones above we must have $r=1$. Since, by (1), we cannot have $p_{1}>2$, it must be that $p_{1}=2$. Also, it is clear that if $n=2^{k}$ then $n=2 \phi(n)$.

Next, we consider convex, rational, equiangular polygons in each of the three cases given by the lemma. For the overdetermined case $(n<2 \phi(n))$ we have the following:

Proposition 3 If $l_{1}, l_{2}, \cdots, l_{p^{k}}$ are the lengths of the edges of a convex, rational, $p^{k}$-gon with $p>2$ prime, then the polygon is equiangular iff

$$
\begin{aligned}
& l_{1}=l_{p^{k-1}+1}=\cdots=l_{(p-1) p^{k-1}+1}, \\
& l_{2}=l_{p^{k-1}+2}=\cdots=l_{(p-1) p^{k-1}+2}, \\
& \vdots \\
& l_{p^{k-1}-1}=l_{2 p^{k-1}-1}=\cdots=l_{p^{k}-1}, \\
& l_{p^{k-1}}=l_{2 p^{k-1}}=\cdots=l_{p \cdot p^{k-1}}=l_{p^{k}} .
\end{aligned}
$$

\section{Proof Let}

$$
\Phi_{n}(X)=1+X^{p^{k-1}}+X^{2 p^{k-1}}+\cdots+X^{(p-1) p^{k-1}}
$$

be the minimal polynomial of $\xi_{n}$ over $\mathbb{Q}[X]$ (see [5], page 31 ). In order to apply Proposition 2, we need to write $\xi_{n}^{j}$, for all $N=\phi\left(p^{k}\right)=(p-1) p^{k-1} \leq j \leq p^{k}-1$, as a linear combination with integer coefficients of $1, \xi_{n}, \xi_{n}^{2}, \cdots, \xi_{n}^{N-1}$. Starting with the equation in $\xi_{n}$ given by its minimal polynomial and multiplying by $\xi_{n}, \xi_{n}^{2}, \cdots, \xi_{n}^{n-N-1}$, we have

$$
\begin{aligned}
& \xi_{n}^{N}=-1-\xi_{n}^{p^{k-1}}-\xi_{n}^{2 p^{k-1}}-\cdots-\xi_{n}^{(p-2) p^{k-1}}, \\
& \xi_{n}^{N+1}=-\xi_{n}-\xi_{n}^{p^{k-1}+1}-\xi_{n}^{2 p^{k-1}+1}-\cdots-\xi_{n}^{(p-2) p^{k-1}+1}, \\
& \quad \vdots \\
& \xi_{n}^{p^{k}-1}=-\xi_{n}^{2 p^{k-1}-1}-\xi_{n}^{3 p^{k-1}-1}-\cdots-\xi_{n}^{(p-1) p^{k-1}-1} .
\end{aligned}
$$

Thus, $a_{i+1, j}=-1$ if $j-(i+1) \in\left\{p^{k-1}, 2 p^{k-1}, \cdots,(p-1) p^{k-1}\right\} \quad$ and $\quad a_{i+1, j}=0$ otherwise. With these values of $a_{i+1, j}$, the conclusion follows.

Consequence 1 Any rational equiangular polygon with a prime number of edges is regular.

Proof This follows based on Observation 1 and the $k=1$ case in Proposition 3.

Observation 3 The consequence above proves conjecture 6 from [4].
For the fully determined case $(n=2 \phi(n))$ we have the following characterization:

Proposition 4 Given a convex, rational polygon whose number of edges is a power of two, the polygon is equiangular iff opposite edges are congruent.

Proof Let $n=2^{k}$ be the number of edges of the polygon. Since $\Phi_{2^{k}}(X)=X^{2^{k-1}}+1$, it follows that $\xi_{n}^{2^{k-1}}=-1, \xi_{n}^{2^{k-1}+1}=-\xi_{n}, \cdots, \xi_{n}^{2^{k}-1}=-\xi_{n}^{2^{k-1}-1}$. Thus, the relation from Theorem 1 becomes

$$
l_{1}+l_{2} \xi_{2^{n}}+\cdots+l_{2^{k-1}-1} \xi_{n}^{2^{k-1}-1}-l_{2^{k-1}}-l_{2^{k-1}+1} \xi_{n}-\cdots-l_{2^{k}} \xi_{n}^{2^{k-1}-1},
$$

or

$$
\left(l_{1}-l_{2^{k-1}}\right)+\left(l_{2}-l_{2^{k-1}+1}\right) \xi_{n}+\cdots+\left(l_{2^{k-1}-1}-l_{2^{k}}\right) \xi_{n}^{2^{k-1}-1}=0 .
$$

But then $\xi_{n}$ is a root of a rational polynomial of degree less than that of $\Phi_{n}$. This is only possible if the polynomial is identically zero, which implies the conclusion.

As a consequence of the proposition above, we obtain a different proof of Theorem 3 from [4].

Consequence 2 There does not exist an equiangular $2^{k}$-gon with integer edge lengths, all distinct.

For the underdetermined case $(n>2 \phi(n))$, given the lack of a simple formula for $\Phi_{n}(X)$ in this case, we will only consider the following example.

Lemma $2 l_{1}, l_{2}, \cdots, l_{15} \in \mathbb{Q}^{+}$are the edge lengths of a convex equiangular 15-gon, with the edges ordered counterclockwise, iff

$$
l_{3}-l_{8}=l_{6}-l_{11}=l_{9}-l_{14}=l_{12}-l_{2}=l_{15}-l_{5}
$$

and

$$
l_{1}-l_{6}=l_{4}-l_{9}=l_{7}-l_{12}=l_{10}-l_{15}=l_{13}-l_{3} .
$$

Proof In this case, $\phi(15)=8$,

$\Phi_{15}(X)=X^{8}-X^{7}+X^{5}-X^{4}+X^{3}-X+1$. By letting $\xi_{15}=\xi$, we have

$$
\begin{gathered}
\xi^{8}=\xi^{7}-\xi^{5}+\xi^{4}-\xi^{3}+\xi_{1}, \xi^{9}=-1+\xi^{2}-\xi^{3}-\xi^{6}+\xi^{7}, \\
\xi^{10}=-1-\xi^{5}, \xi^{11}=-\xi-\xi^{6}, \xi^{12}=-\xi^{2}-\xi^{7}, \\
\xi^{13}=1-\xi-\xi^{4}+\xi^{5}-\xi^{7} .
\end{gathered}
$$

Based on these relations and Proposition 2, we must have

$$
\begin{gathered}
l_{1}-l_{9}-l_{10}-l_{11}+l_{14}+l_{15}=0, l_{2}+l_{9}-l_{12}-l_{14}=0, \\
l_{3}+l_{10}-l_{13}-l_{15}=0, l_{4}-l_{9}-l_{10}+l_{15}=0, \\
l_{5}+l_{9}-l_{14}-l_{15}=0, l_{6}-l_{9}-l_{11}+l_{14}=0, \\
l_{7}-l_{10}-l_{12}+l_{15}=0, l_{8}+l_{9}+l_{10}-l_{13}-l_{14}-l_{15}=0 .
\end{gathered}
$$

If we let

$$
\begin{gathered}
l_{3}-l_{8}=c_{1}, l_{6}-l_{11}=c_{2}, l_{9}-l_{14}=c_{3}, \\
l_{12}-l_{2}=c_{4}, l_{15}-l_{5}=c_{5}
\end{gathered}
$$


and

$$
\begin{gathered}
l_{1}-l_{6}=d_{1}, l_{4}-l_{9}=d_{2}, l_{7}-l_{12}=d_{3}, \\
l_{10}-l_{15}=d_{4}, l_{13}-l_{3}=d_{5},
\end{gathered}
$$

the relations above become

$$
\begin{aligned}
& d_{1}+c_{2}-c_{3}-d_{4}=0, c_{3}=c_{4}, d_{4}=d_{5}, d_{2}=d_{4}, \\
& c_{5}=c_{3}, c_{2}=c_{3}, d_{3}=d_{4},-c_{1}-d_{5}+d_{4}+c_{3}=0 .
\end{aligned}
$$

Clearly, these relations are equivalent to

$c_{1}=c_{2}=c_{3}=c_{4}=c_{5}$ and $d_{1}=d_{2}=d_{3}=d_{4}=d_{5}$, thus proving the lemma.

\section{Arithmetic Polygons}

Following the terminology from [4], a polygon is said to be arithmetic if it is equiangular and its edge lengths (in some order) form a nontrivial arithmetic sequence. As shown in the same paper, an arithmetic $n$-gon exists iff there exists an equiangular polygon with edge lengths (in some order) $1,2, \cdots, n$. In this section we find a necessary and sufficient condition for the existence of arithmetic polygons in terms of the number of edges. First, we have the following:

Consequence 3 There are no arithmetic polygons whose number of edges is the power of a prime.

Proof This follows as a consequence of Propositions 3, 4, and Observation 1.

One case when arithmetic polygons do exist is provided by the example below.

Example 1 There exists a (convex) arithmetic 15-gon. Proof If we select

$$
\begin{aligned}
& l_{1}=15, l_{2}=7, l_{3}=4, l_{4}=11, l_{5}=8, l_{6}=5, l_{7}=12, l_{8}=9, \\
& l_{9}=1, l_{10}=13, l_{11}=10, l_{12}=2, l_{13}=14, l_{14}=6, l_{15}=3,
\end{aligned}
$$

then the conditions in Example 2 are satisfied since

$$
l_{3}-l_{8}=l_{6}-l_{11}=l_{9}-l_{14}=l_{12}-l_{2}=l_{15}-l_{5}=-5
$$

and

$$
l_{1}-l_{6}=l_{4}-l_{9}=l_{7}-l_{12}=l_{10}-l_{15}=l_{13}-l_{3}=10 .
$$

Observation 4 The proposition above provides a counterexample to conjecture 7 from [4] claiming that no arithmetic $n$-gons exist if $n$ is odd.

The example above suggests the following:

Theorem 2 There exists an arithmetic n-gon if and only if $n$ is not the power of a prime, i.e., $n$ has at least two distinct primes factors.

Proof By Consequence 3, it is enough to prove the converse. So, let's consider $n=p q$, for some positive integers $p, q$. Since $n$ is not the power of a prime, $p$ and $\mathrm{q}$ can be chosen to be relatively prime. If $\xi$ denotes a primitive $(p q)$-th root of unity, then

$$
1+\xi^{p}+\xi^{2 p}+\cdots+\xi^{(q-1) p}=0
$$

and

$$
1+\xi^{q}+\xi^{2 q}+\xi^{(p-1) q}
$$

Multiplying relations (1.6) by $a \xi^{a}, 1 \leq a \leq p$ and (1.7) by $(b-1) p \xi^{b}, 1 \leq b, \leq q$ we have

$$
\sum_{k=0}^{q-1} a \xi^{a+k p}=0
$$

and

$$
\sum_{l=0}^{p-1}(b-1) p \xi^{b+l q}=0 .
$$

Let us now observe that every integer between 1 and $p q$ appears exactly once as an exponent in both (1.8) and (1.9) due to the fact that $p$ and $q$ are relatively prime. If we add all $p$ equations (1.8) and all $q$ equations (1.9), we obtain

$$
\sum_{a=1}^{p} \sum_{k=0}^{q-1} a \xi^{a+k p}+\sum_{b=1}^{q} \sum_{l=0}^{p-1}(b-1) p \xi^{b+l q}=0
$$

Whenever $a+k p=b+l q$, the sum of the corresponding coefficients $a+(b-1) p$ is an integer between 1 and $p q$ Moreover, different $a$ and $b$ with $1 \leq a \leq p$, $1 \leq b \leq q$, generate different values for $a+(b-1) p$ because $p$ and $q$ are relatively prime. Since there are exactly $p q$ pairs $(a, b)$, the values of $a+(b-1) p$ will represent a permutation of the set $\{1,2, \cdots, p q\}$.

\section{Conclusions}

In this note we determined all rational equiangular polygons whose number of sides a prime power. Although we also determined all rational equiangular 15-gons, the general problem remains open. In addition, we provided a complete characterization of arithmetic polygons.

As an interesting application, we note that, as mentioned in [6], there is a nice correspondence arising from the Schwarz-Christoffel transformations between equiangular $n$-gons and certain areas determined by binary forms of degree $n$ with complete factorizations over $\mathbb{R}$. It would be interesting to investigate the consequences of our results in the language of binary forms.

\section{REFERENCES}

[1] D. Ball, "Equiangular Polygons," The Mathematical Gazette, Vol. 86, No. 507, 2002, pp. 396-407. http://dx.doi.org/10.2307/3621131

[2] P. R. Scott, "Equiangular Lattice Polygons and Semiregular Lattice Polyhedra," College Mathematics Journal, Vol. 18, No. 4, 1987, pp. 300-306. http://dx.doi.org/10.2307/2686799

[3] R. Honsberger, "Mathematical Diamonds," The Mathematical Association of America, Washington DC, 2003.

[4] R. Dawson, “Arithmetic Polygons,” American Mathe- 
matical Monthly, Vol. 119, No. 8, 2012, pp. 695-698. http://dx.doi.org/10.4169/amer.math.monthly.119.08.695

[5] P. Samuel, “Algebraic Theory of Numbers,” Kershaw, 1972.

[6] M. A. Bean, "Binary Forms, Hypergeometric Functions and the Schwarz-Christoffel Mapping Formula,” Trans- actions of the American Mathematical Society, Vol. 347, No. 12, 1995, pp. 4959-4983.

http://dx.doi.org/10.1090/S0002-9947-1995-1307999-2 